\title{
Nordiques
}

$37 \mid 2019$

Mythes et réalités de l'Arctique

\section{L'imaginaire viking et les extrêmes droites française et belge contemporaines}

\section{Stéphane François}

\section{(2) OpenEdition \\ 12 Journals}

Édition électronique

URL : http://journals.openedition.org/nordiques/464

DOI : $10.4000 /$ nordiques.464

ISSN : $2777-8479$

Éditeur :

Association Norden, Bibliothèque de Caen la mer

\section{Édition imprimée}

Date de publication : 1 mai 2019

Pagination : 113-130

ISBN : 979-1-0959140-3-7

ISSN : 1761-7677

\section{Référence électronique}

Stéphane François, "L'imaginaire viking et les extrêmes droites française et belge contemporaines », Nordiques [En ligne], 37 | 2019, mis en ligne le 31 octobre 2020, consulté le 13 mars 2021. URL : http:// journals.openedition.org/nordiques/464 ; DOI : https://doi.org/10.4000/nordiques.464 


\section{L'imaginaire viking et les extrêmes droites française et belge contemporaines}

Stéphane François*

\section{RÉSUMÉ}

Les marges les plus radicales des extrêmes droites française et belge se sont intéressées, à compter des années 1970, à la spiritualité des Vikings. Cet intérêt sest concrétisé par la création, à cette époque, de pratiques à la fois cultuelles et sociales (rites de mariage, de mort, célébration des solstices et de Noël, etc.). Cet attrait a été dynamique entre les années 1970 et le début des années 2010. Venue des marges les plus radicales (le milieu des anciens nazis), cette spiritualité sest diffusée dans d'autres tendances de l'extrême droite, proche des premières, comme celles des nouvelles droites et de l'identitarisme. Après avoir expliqué notre méthodologie, nous proposons de revenir sur la généalogie de cet intérêt, de montrer le rôle de ces pratiques pour ces groupes, en lien avec un ethnocentrisme assumé.

ABSTRACT

The most radical margins of the French and Belgian far right were interested, from the 1970s, to the spirituality of the Vikings. This interest was concretized by the creation, at that time, of cultural and social practices (rites of marriage, death, celebration of solstices and Christmas, etc.). This attraction was dynamic between the 1970s and the beginning of the year 2010. From the most radical margins (the ex nazis' milieu), this spirituality has spread in other far-right tendencies, close to the former, like those New Rights and Identitarianism. After explaining our methodology, we propose, in this article, to return to the genealogy of this interest, to show the role, for these groups, of these practices, in connection with an assumed ethnocentrism.

\footnotetext{
* Docteur en sciences politiques, membre associé du GSRL (EPHE/CNRS/PSL).
} 
L'extrême droite européenne, voire euro-américaine - connue aujourd'hui sous l'expression d' " alt-right $"^{1}-$, s'est passionnée pour le monde viking au sortir de la Seconde Guerre mondiale. Il s'agissait à la fois de trouver un palliatif à la thématique aryenne, trop connotée "nazie ", tout en gardant l'idée d'une origine polaire de la civilisation blanche, et un moyen d'élaborer une nouvelle spiritualité européenne, c'est-à-dire néopaïenne ${ }^{2}$ ne devant rien aux religions monothéistes. Ainsi, le militant identitaire belge Bernard Mengal ${ }^{3}$ affirme, vers 1993-1994 - la publication dans laquelle il s'exprime n'étant pas datée : "Toutes les œuvres nordiques démontrent que les univers européen et hébraïque sont si différents qu'on peut parler d'une profonde incompatibilité et d'une incompréhension réciproque. ${ }^{4}$ Cette assertion est importante pour notre propos, car elle est le cœur du militantisme païen-nordique d'extrême droite pour trois raisons. Ces militants veulent : 1/une spiritualité qui ne doive rien au monothéisme; 2/qui soit enracinée (avec le mythe de l'origine polaire des Indo-Européens ${ }^{5}$ ) ; 3/qui puisse continuer à exprimer un antisémitisme discret (l'incompatibilité du judaïsme avec les valeurs « enracinées » européennes) ou ostensible ${ }^{6}$.

L'objectif de cet article sera de montrer le rôle de cette vision de la spiritualité viking dans la cohésion du groupe et dans la volonté de créer une vision du monde cohérente ; en ce sens, il s'agira de faire une mise au point sur une nouvelle étape de la circulation de l'imaginaire viking dans les milieux néopaïens d'extrême

1 Stéphane François, "Qu’est-ce que l'alt-right ? ", Paris, Fondation Jean Jaurès, 2017, [https://jeanjaures.org/nos-productions/qu-est-ce-que-l-alt-right].

2 Cependant, l'extrême droite ne fut pas la seule à vouloir réactiver la foi des Vikings. Ainsi, en Islande, la version islandaise du paganisme nordique, l'Ásatrú, est devenue religion officielle dès 1973. Depuis cette date, il connaît une forte croissance annuelle. Plus récemment, la Grande-Bretagne a vu, dans les années 1990, la nomination d'aumôniers païens dans ses universités, notamment à Leeds. En outre, il ne faut pas oublier que le prince de Galles est traditionnellement Grand Druide. Cette mode païenne a même gagné le très protestant Danemark où le culte païen des Vikings a été reconnu religion officielle le 6 novembre 2003.

3 Nous étudions son parcours et son militantisme infra.

4 «Entretien avec Bernard Mengal ", Hammer Against Cross, no 3, s. d., p. 15.

5 Stéphane François, Au-delà des vents du Nord. L'extrême droite française, le pôle Nord et les IndoEuropéens, Lyon, Presses universitaires de Lyon, 2014.

6 Le néopaganisme nordique dans sa variante extrémiste de droite fait partie de l'idéologie néonazie et néo-völkisch. Son fondement est l'idée d'une religion du sang et du sol, c'est-à-dire une religion ethnique enracinée. L'antisémitisme y est enraciné. L'influence des thèses völkisch est d'ailleurs flagrante. Aprèsguerre, les ex-SS qui reprirent l'activisme politique transmirent cette foi aux jeunes générations. Ainsi, on voit la participation de plusieurs SS, Allemands et Français, aux activités de la Nouvelle Droite entre les années 1970 et le milieu des années 1980. Aujourd'hui, elle reste une composante majeure des milieux identitaires, néonazis et suprémacistes blancs. Dans les années 2000, lorsque vous demandiez, dans les milieux des librairies d'extrême droite, des livres "païens ", le libraire vous montrait systématiquement le rayon nazi/néonazi. Nous en avons fait plusieurs fois l'expérience dans des librairies parisiennes (La Licorne Bleue, Primatice et La Librairie du Savoir - ouvertement négationniste), lors de la constitution de notre documentation pour l'écriture de notre thèse (Les néo-paganismes et la Nouvelle Droite (1980-2006). Pour une autre approche - paru en 2008 chez Archè-Milan -) et de l'ouvrage intitulé Au-delà des vents du Nord. L'extrême droite française, le pôle Nord et les Indo-Européens. 
droite. Il s'agira aussi d'en montrer les récentes évolutions. Pour ce faire, il s'articulera en quatre points : le premier explicitera notre méthodologie ; le deuxième reviendra sur l'histoire récente du néopaganisme d'extrême droite en France et en Belgique ; le troisième portera sur ses expressions sociales internes aux groupes et le dernier, enfin, montrera la persistance d'un ethnocentrisme très fort dans ces milieux.

\section{Méthodologie}

Le contenu portera ici principalement sur la période 1970-2000. Ce choix chronologique est motivé par :

Premièrement, il correspond à une évolution doctrinale et spirituelle importante de l'extrême droite occidentale : le néopaganisme ${ }^{7}$, longtemps confiné aux sphères néonazies, trouve de nouveaux adeptes dans d'autres milieux de l'extrême droite. Il devient alors l'une des spiritualités importantes de l'extrême droite à partir de la seconde moitié des années $1980^{\circ}$. En France, il naît dans les milieux allant des anciens SS et se développe dans le cadre du Groupe de recherches et d'études de la civilisation européenne (GRECE) ${ }^{9}$, qui théorisera entre 1968 et le milieu des années 1980 des formes de néopaganisme politique, marquées dans un premier temps par le matérialisme biologique ${ }^{10}$. Le GRECE est une école de cadres politiques d'extrême droite fondée en 1968, de tendance nationaleeuropéenne, ayant abandonné le militantisme politique, jugé inefficace. Sa stratégie porte sur l'entrisme et la diffusion de ces idées dans les différents secteurs de la société (le "gramscisme de droite "). Il est connu depuis 1979 sous l'expression "Nouvelle Droite ». Son discours a beaucoup évolué dans le temps. Aujourd'hui, il se caractérise par un anticapitalisme, un anti-américanisme, un ethnodifférentialisme radical, un européisme, un écologisme, une promotion d'une forme de paganisme philosophique. Il conçoit les identités comme des constructions

7 Pour une définition du néopaganisme, voir Stéphane François, "Néopaganisme ", in Dictionnaire des Barbares, B. Dumézil (dir.), Paris, PUF, 2016, p. 970-973.

8 Aux États-Unis, le néopaganisme d'extrême droite dépasse en nombre et en influence les groupuscules nazis locaux, ainsi que les franges radicales du Ku Klux Klan. En 2003, environ 50 \% des adeptes américains du paganisme nordique, réinventant donc la religion des Vikings, sont des extrémistes de droite, en particulier en prison, promouvant la supériorité de la race aryenne, l'antisémitisme, le racisme et le négationnisme (Matthias Gardell, Gods of the Blood. The Pagan Revival and White Separatism, Londres - Durham, Duke University Press, 2003). Depuis, ce chiffre a continué d'augmenter, pour se situer actuellement aux alentours de $60 \%$ des adeptes.

9 Sur la Nouvelle Droite, voir Pierre-André Taguieff, Sur la Nouvelle Droite. Jalons d'une analyse critique, Paris, Descartes \& Cie, 1994 ; Stéphane François, Les néo-paganismes et la Nouvelle Droite (19802006). Pour une autre approche, Milan, Archè-Milan, 2008 ; Stéphane François, Au-delà des vents du Nord. L'extrême droite française, le Pôle nord et les Indo-Européens, Lyon, Presses universitaires de Lyon, 2014.

10 Sur les filiations entre le national-socialisme et le GRECE, voir Stéphane François, «La Nouvelle Droite et le nazisme. Retour sur un débat historiographique ", Revue française d'histoire des idées politiques, no 46, 2017, p. 93-115. 


\section{ethnoculturelles et défend l'idée d'une identité européenne héritée des peuples} indo-européens de la protohistoire. Son principal théoricien est Alain de Benoist.

\section{La jonction entre les premiers et les seconds se fait via l'intermédiaire de} l'écrivain, journaliste et militant régionaliste, "socialiste-européen ${ }^{11}$ - pour reprendre une expression très prisée dans ces milieux, forgée durant la Seconde Guerre mondiale par les partisans du Nouvel ordre européen - et surtout völkisch $^{12}$, Jean Mabire ${ }^{13}$ qui édita dès 1949 , et jusqu'en 1958, une revue intitulée Viking, devenue culte dans les milieux de l'extrême droite ${ }^{14}$. Mabire était un écrivain prolifique, touche-à-tout : il écrivit aussi bien sur l'histoire militaire, avec un

11 Sur les idées de Mabire, voir Nicolas Lebourg et Jonathan Preda, «Le Front de l'Est et l'extrême droite radicale française : propagande collaborationniste, lieu de mémoire et fabrique idéologique ", in Références et thèmes des droites radicales, O. Dard (dir.), Berne, P. Lang, 2015, p. 101-138.

12 Ce courant est une forme de racialisme plus ou moins néopaïn présent en Allemagne et en Autriche durant la seconde moitié du XIX" siècle. Le terme «völkisch», réputé intraduisible en français, l'est souvent par « raciste ». La racine "Volk " signifie " peuple ", mais son sens va au-delà de celui de "populaire ", dans une acception foncièrement ethnique. La meilleure traduction serait « ethnonationalisme ». Il peut être compris comme nostalgie folklorique et raciste d'une préhistoire allemande largement mythifiée. Ce courant bigarré puisait ses références dans le romantisme, dans l'occultisme, dans les premières doctrines "alternatives " (médecines douces, naturisme, végétarisme, etc.) et enfin dans les doctrines racistes. La reconstitution d'un passé germanique largement mythique a éloigné les Völkische des religions monothéistes pour tenter de recréer une religion païenne, purement allemande. Toutefois, de nombreux Völkische restent des chrétiens croyants. Enfin, il existe des Völkische " politiques » n'ayant que faire des spéculations religieuses ou spirituelles. Ce mouvement est une nébuleuse d'organisations tenantes d'un nationalisme biologisé et irrédentiste partageant, outre ce nationalisme particulier, trois fondements pratiquement invariables : 1/un antisémitisme presque unanime; $2 /$ un projet de révolution élitaire renversant la république de Weimar ; 3/la mise en place d'une politique révisionniste de restauration politique et impériale. Voir Francis Bertin, "Ésotérisme et vision de la race dans le courant "völkisch" ", Politica Hermetica, n 2, 1988, p. 83-92; Louis Dupeux, Histoire culturelle de l'Allemagne 1919-1960, Paris, PUF, 1989, p. 51-54 ; Jean-Pierre Faye, Langages totalitaires. Précédé de Théorie du récit, Paris, Hermann, 1973, p. 151-199 ; Uwe Puschner, Die völkische Bewegung im wilhelminischen Kaiserreich, Sprache, Rasse, Religion, Darmstadt, Wissenschaftliche Buchgesellschaft, 2001 ; Antisemitismus, Paganismus, völkische Religion, H. Cancik et U. Puschner (dir.), Munich, K. G. Saur, 2004. Nous renvoyons aussi le lecteur vers l'étude de Sabine Doering-Manteuffel, L'occulte. Histoire d'un succès à l'ombre des Lumières. De Gutenberg au World Wide Web, Paris, Éditions de la Maison des sciences de l'homme, 2011, en particulier le chapitre intitulé "Monde primitif et univers. L'occultisme national-populiste dans les théories de la globalité au $\mathrm{XX}^{\mathrm{e}}$ siècle », p. $162-189$ et 268.

13 Jean Mabire (1927-2006) est un vieux militant de l'extrême droite française. Un militantisme commencé dès 1949 avec la création de la revue régionaliste normande Viking. Officier parachutiste durant la guerre d'Algérie, il devient ensuite journaliste. Il participe à Défense de l'Occident du néofasciste Maurice Bardèche et Salut public - pro-Algérie française. Il fait partie de l'équipe d'Europe-Action, la revue et le groupe éponyme de Dominique Venner, dont il deviendra le rédacteur en chef. Il fait partie ensuite de l'équipe du GRECE, intégrant l'équipe du magazine Éléments. Au milieu des années 1980, il quitte le GRECE pour rejoindre le FN, qu'il quittera également pour suivre la scission mégrétiste. En 1995, il fonde le groupuscule Terre \& Peuple avec les universitaires néodroitiers Pierre Vial et Jean Haudry. Les thèses de Jean Mabire sur l'Europe des ethnies ont eu une forte influence sur les militants d'extrême droite.

14 La revue fut rééditée en deux volumes de 511 et 592 pages, tirés à 250 exemplaires, en 1999, par la maison d'édition Le Veilleur de Proue. 
net tropisme pour la " geste " de la $\mathrm{SS}^{15}$, sur la littérature et les écrivains - il était critique littéraire $^{16}$-, que sur le monde nordique ${ }^{17}$ ou le néopaganisme ${ }^{18}$.

Deuxièmement, cette période est riche en publications internes et externes aux différents groupes, pour la plupart très confidentielles, telles les revues citées dans cet article. La collecte de ces publications a été longue et parfois fastidieuse : nous sommes passés par les réseaux spécialisés, assez "underground " sites de vente en ligne, librairies nationalistes, chez les auteurs, etc. En effet, les revues citées ici sont confidentielles : à l'exception de revues comme Éléments, disponible en kiosque, certaines d'entre elles ne sont que des fanzines photocopiés, disponibles uniquement chez l'auteur. C'est le cas par exemple de Hammer against Cross au titre explicite ( Le Marteau contre la Croix »), un fanzine néopaïen d'extrême droite des années 1990, dont les numéros ont été achetés dans une boutique de musiques indépendantes ; de la luxueuse revue néopaïenne - hors commerce Irmin reçue directement de son responsable ; de la revue militante bruxelloise Megin et de son Gjallarhorn, du fanzine Omega piloté par des militants nationalistes-révolutionnaires proches du groupuscule Résistance, dont certains dirigeants étaient des compagnons de route du GRECE et dont les numéros ont été achetés dans une librairie d'extrême droite parisienne où ils étaient en dépôt, ou enfin de Ialon, une revue néopaïenne bretonne, proche idéologiquement de la Nouvelle Droite, émanation d'un groupuscule, la Kredenn Geltiek Hollvedel, lui-même héritier d'un groupe fondé en 1936 par le militant régionaliste breton Morvan Marchal. Nous avons acheté les numéros directement auprès du responsable du groupuscule.

15 Il a ainsi publié sept ouvrages sur la SS, entre 1972 et 1996 : Les Waffen SS, Paris, Balland, 1972 (sous le pseudonyme d'Henri Landemer) ; La brigade Frankreich, Paris, Fayard, 1973 ; La division Charlemagne, Paris, Fayard, 1974 ; Mourir à Berlin, Paris, Fayard, 1975 ; Les jeunes fauves du Führer. La division SS Hitlerjugend en Normandie, Paris, Fayard, 1976 ; La division Wiking, Paris, Fayard, 1980 ; La division de choc Wallonie. Lutte à mort en Poméranie, Paris, Grancher, 1996.

16 Ses critiques ont été réunies dans la série des Que lire ?, 7 volumes parus à ce jour, chez plusieurs éditeurs : Éditions de National-Hebdo, Irminsul et enfin chez Dualpha.

17 Benoît Marpeau, "Le rêve nordique de Jean Mabire ", Annales de Normandie, 43e année, no 3, 1993, p. 215-241. Il a publié notamment les livres suivants sur le sujet (la liste est non exhaustive) : Jean Mabire, Les Vikings, rois des tempêtes, Genève, Idégraf, 1978, et du même auteur ; Les dieux maudits. Récits de mythologie nordique, Paris, Copernic, 1978 ; Les Vikings en Normandie, Paris, Copernic, 1979 ; La saga de Godefroy le Boiteux, Paris, Copernic, 1980 ; Les grands aventuriers de l'histoire. Les éveilleurs de peuples, Paris, Fayard, 1982 ; Guillaume le Conquérant, Art et Histoire d'Europe, 1987 ; Les grands aventuriers de l'histoire, Paris, Fayard, 1982 ; Les Vikings à travers le monde, Louviers, L'Ancre de marine, 1992 ; Légendes de la mythologie nordique, Rennes, Ouest France, 1995 ; Légendes traditionnelles de Normandie, Louviers, L'Ancre de marine, 2001 ; Jean Mabire, Jean-Robert Ragache, Histoire secrète de la Normandie, Paris, France Empire, 1986. Voir la bibliographie établie par Alain de Benoist pour le compte de l'Association des amis de Jean Mabire, Bibliographie de Jean Mabire, Pont-Authou, Héligoland, 2010.

18 Jean Mabire, Thulé, le soleil retrouvé des Hyperboréens, Paris, R. Laffont, 1978 ; Les solstices. Histoire et actualité, en collaboration avec Pierre Vial, Paris, GRECE, 1975 ; Les dieux maudits, Paris, Copernic, 1978 . 
Cependant, nous devons reconnaître que depuis les années 2010, ce néopaganisme est plus discret en France. Il est toujours pratiqué, mais certains groupes, comme Les Identitaires (anciennement le Bloc Identitaire), le minimisent afin d'attirer de nouveaux militants, parfois rebutés par cet aspect, car souvent de confession catholique. Enfin, certains groupes étant sur le déclin, ou ayant implosé, la période 2010 jusqu'à aujourd'hui correspond à un moment de recomposition. Il est donc nécessaire de prendre du recul afin de pouvoir avoir une vision élargie des évolutions en cours. En outre, afin de comprendre l'origine et l'essor de ce néopaganisme français et belge, il est nécessaire de faire un état des lieux du paganisme viking d'extrême droite en France et en Belgique en prenant quelques exemples récents.

\section{DE L'APRÈS-GUERRE AU DÉBUT DES ANNÉES 2000}

L'extrême droite de l'après Seconde Guerre mondiale s'est intéressée aux Vikings pour la spiritualité. Reprenant l'héritage des groupes völkisch et aryosophes $^{19}$ d'avant-guerre ${ }^{20}$, ces militants d'extrême droite estiment que la proximité du cercle polaire rendrait les Vikings plus " purs ", plus européens, spirituellement et physiquement. Plus le militant est proche du Nord, plus il serait en contact avec une pureté spirituelle propre aux Indo-Européens, ces derniers ayant une origine polaire ${ }^{21}$. De ce fait, une majorité des néopaïens d'extrême droite pratiquent une foi d'inspiration nordico-germanique. Le néopaganisme nordique, reconstruit à partir de la religion des Vikings, dont certaines variantes contemporaines sont connues sous les noms d'odinisme ou d'Ásatrú, est l'un des néopaganismes qui attirent le plus les militants d'extrême droite ${ }^{22}$. En France, c'est la Nouvelle Droite qui en fit la promotion ${ }^{23}$. Cela pour des raisons aisément compréhensibles : le type physique nordique y est souvent vu comme l'archétype ethnique de l'Européen. Selon ces néopaïens, le domaine ethnoculturel nordique va de la Scandinavie à la France (qui serait de population franque et burgonde, présence viking en Normandie) et du monde germanique aux îles Britanniques, en passant par le Benelux (avec les Frisons).

19 Sur l'histoire des groupes aryosophes autrichiens, voir les travaux de l'historien britannique Nicholas Goodrick-Clarke, The Occult Roots of Nazism. Secret Aryan Cults and their Influence on Nazi Ideology, New York, New York University Press, 1985.

20 Voir par exemple la traduction en 2013 aux éditions de l'Homme libre, un éditeur néonazi fondé par un ancien responsable du GUD (Groupe union défense), William Bonnefoy, du livre de Guido von List, Le secret des runes.

21 Stéphane François, Au-delà des vents du Nord, op. cit.

22 Matthias Gardell, Gods of the Blood. The Pagan Revival and White Separatism, op. cit.

23 Voir Stéphane François, Les néo-paganismes et la Nouvelle Droite (1980-2006), op. cit. 
Viking, la revue normande de l'écrivain "folkiste " ${ }^{24}$ Jean Mabire, publiée de 1949 à 1958, peut être considérée comme la pionnière en France dans la diffusion de ces thèmes. Ceux-ci portaient à la fois sur l'exaltation de la santé physique (sport, critique de l'alcool et du tabagisme, hygiène) et sur la culture populaire norroise. Le ton y était ouvertement païen. D’ailleurs, Mabire se définissait comme un adepte de l'odinisme ${ }^{25}$ depuis cette époque, sans pour autant faire partie d'une structure précise. Cette revue fit par la suite des émules ${ }^{26}$. Ainsi, une autre publication normande, Heimdal, fondée en 1971 et héritière de Viking ${ }^{27}$, a joué un rôle important dans la diffusion de l'intérêt pour le monde scandinave. Son contenu était proche des thèmes de la Nouvelle Droite. Georges Bernages, son fondateur, s'était d'ailleurs associé aux éditions Copernic, la maison d'édition du GRECE en 1979. Elle cessa de paraître à la fin des années 1980 pour se transformer en éditions Heimdal, qui publient des ouvrages sur la Normandie et la Seconde Guerre mondiale. Au début des années 1970, la revue Heimdal organisait des célébrations du solstice d'été. Ainsi, en 1974 : «Une centaine d'adhérents parisiens du GRECE ont participé, le samedi 22 juin, au solstice organisé à Falaise par la revue Heimdal et par la Fédération culturelle norroise. Placé sous le patronage du $\mathrm{D}^{\mathrm{r}}$ Paul German, maire de Falaise, réalisé avec la collaboration du comité des fêtes de la ville et du groupe folklorique La Mesnie-Guillaume, ce solstice a réuni plus de 1500 personnes dans l'enceinte du château de Guillaume le Conquérant. ${ }^{28}$ Le $\mathrm{D}^{\mathrm{r}}$ German, maire divers droite de la ville de Falaise et ancien président du conseil régional de Basse-Normandie de 1978 à 1982, était un ancien président du Mouvement normand dont a également fait partie Jean Mabire. Les thèses païennes de Jean Mabire sur l'Europe des ethnies ont eu une forte influence sur les militants d'extrême droite des années 1970 à nos jours.

Dans les années 1990, le groupe odiniste le plus important dans notre pays était l'IRMIN, ou Institut de recherche sur la mythologie et l'identité nordique, émanation de militants de la Nouvelle Droite. Il publiait une revue éponyme. L'IRMIN « ... a pour objet d'étudier tous les aspects de la tradition nordique anti-chrétienne (germanique et scandinave) en les comparant aux autres traditions religieuses ou spirituelles européennes (celtiques, slaves, gréco-romaines), chrétiennes et orientales (zoroastrisme, bouddhisme, hindouisme, shintoïsme). Il ne se considère pas comme un mouvement religieux puisque son activité est

24 Pour l'usage et la pertinence du néologisme " folkiste », voir mes travaux, notamment, "L'extrême droite "folkiste" et l'antisémitisme ", Le Banquet, no 24, 2007, p. 255-269 et Au-delà des vents du Nord, op. cit.

25 Jean Mabire, «Héligoland. Île centre et île sacrée du monde hyperboréen », in Collectif, Balades au cour de l'Europe païenne, Saint-Jean-des-Vignes, Éditions de la Forêt, 2002, p. 39.

26 Nous pouvons citer par exemple la revue Balder, fondée par le journaliste Emmanuel Ratier (19572015), qu'il dirigea de 1976 à 1979. Il était aussi un ancien membre du GRECE et un païen déclaré.

27 Benoît Marpeau, "Le rêve nordique de Jean Mabire ", op. cit.

28 Non signé, "La fête du solstice du 22 juin 1974 ", Eléments, no 6, 1974, p. 3-4. 
axée sur la recherche ; il n'impose pas de dogme, laissant chacun libre de croire quelque chose ou pas. [...] L'association est structurée en deux unités de travail : "mythologie" et "identité", correspondant aux deux aspects de l'intitulé de l'association. Elles portent les noms des deux corbeaux d'Odin : Munin ("la mémoire" $[\ldots]$ ) et Hugin ("la conscience" [...]) $»^{29}$. Le siège de l'association était, durant une période, situé à Reims, lieu de vie de son fondateur, Arnaud d'Apremont. La présidente de l'association était Anne-Laure d'Apremont, et le président d'honneur l'universitaire Paul-Georges Sansonetti ${ }^{30}$.

Arnaud d'Apremont est l'ancien rédacteur en chef de la revue Secrets et Sociétés, une revue conspirationniste fondée par Philippe Randa ${ }^{31}$. Il a aussi collaboré à Heimdal et fut enfin directeur de collection aux éditions Pardès. Adhérent durant un temps du GRECE, il s'occupait de l'Association des amis de la revue Éléments (AREL). Son ex-épouse, Anne-Laure d'Apremont, a, quant à elle, été proche du Front national de la jeunesse, puis de Troisième Voie. Contrairement à son exépouse, Arnaud d'Apremont était peu militant ${ }^{32}$. Il ne se serait d'ailleurs intéressé au GRECE qu'au travers du néopaganisme. Par la suite, il s'est rapidement éloigné des milieux extrémistes de droite. Le couple d'Apremont est connu pour la qualité de leurs traductions de livres odinistes, notamment ceux de militants païns, comme le Britannique Nigel Pennick, Edred Thorsson (pseudonyme de l'universitaire d'extrême droite états-unien Stephen Flowers) ou de la magicienne néerlandaise Freya Aswynn. Ils ont aussi publié plusieurs textes sur ces thèmes ${ }^{33}$.

Arnaud d'Apremont, dans un entretien accordé en 1995 à un fanzine du nom d'Oméga, définit IRMIN comme un mouvement areligieux qui accepte les personnes quelle que soit leur religion. Toutefois, il précise aussitôt : " Mais il est évident que les sujets que nous investiguons peuvent aisément déboucher sur une pratique religieuse, et plus précisément sur une pratique religieuse traditionnelle

29 "Présentation d'Irmin ", Ialon, no 5, 1993, p. 57.

30 Paul-Georges Sansonetti est un spécialiste de la littérature comparée, qu'il analyse d'un point de vue ésotérique. Il a été chargé de cours à l'École pratique des hautes études. Il est proche des tendances ésotérique et néopaïenne de la Nouvelle Droite. Ainsi, il fait partie de la revue Hyperborée, aux côtés d'autres païens néodroitiers historiques comme Pierre-Émile Blairon, Jean Haudry ou Guillaume Faye. Il est un traditionaliste néodroitier, proche des milieux nordicistes. Son intérêt pour ces théories est ancien : il y a consacré en 1998 un long article, "Le thème du foyer originel dans la tradition scandinave ", paru dans Antaïos (no 13, 1998, p. 134-146) dans lequel il insiste sur l'origine hyperboréenne de la tradition indo-européenne.

31 Philippe Randa, pseudonyme de Philippe-André Dusquene est un journaliste, écrivain et éditeur français, proche des nationalistes-révolutionnaires (c'est un ancien membre du GUD et du Parti des forces nouvelles) et du GRECE. Il a créé trois maisons d'édition (Dualpha, Déterna, L'Æncre) qui existent toujours. En 2016, il a fondé le site Internet Eurolibertés, qu'il dirige.

32 Voir Stéphane François, Les néo-paganismes et la Nouvelle Droite (1980-2006), op. cit., p. 136-137.

33 Anne-Laure et Arnaud d'Apremont, Runes, Puiseaux, Pardès, 1997 et Tradition nordique, Puiseaux, Pardès, 1999, 2 vol. ; Arnaud d'Apremont, Père Noël, Puiseaux, Pardès, 1999. 
européenne c'est-à-dire païenne. " $^{34}$ Il reconnaît aussi que l'IRMIN a des liens avec une grosse structure odiniste britannique, l'Odinic Rite : " ... nous avons décidé de l'aider notamment en lui offrant de partager notre boîte postale et de lui ouvrir les colonnes de notre lettre d'information mensuelle ". Cependant, " la majorité des membres de l'IRMIN ne sont pas membres de l'Odinic Rite et tous les membres de l'Odinic Rite ne sont pas membres de l'IRMIN ${ }^{35}$. Arnaud d'Apremont a animé jusqu'au début des années 2000 son groupe odiniste qu'il a tenu à l'écart de toute idéologie politique, avant de s'éloigner définitivement de ce militantisme.

Il a enfin existé une autre structure importante néopaïenne néodroitière, fondée par le Belge Bernard Mengal, un vieux militant de l'extrême droite, très proche des idéaux völkisch. Il s'agit de l'Association des successeurs des Ases (ASA), active depuis 1992, plus connue sous le nom des Fils des Ases. Elle publie la revue bimestrielle Megin (" pouvoir ") dont le sous-titre est "Organe de combat et de réflexion des Fils des Ases ». Megin a pris la suite de Combat païen, fondé en 1989 par Mengal et Robert Steuckers ${ }^{36}$. Bernard Mengal est aussi l'auteur d'un Manifeste pour un paganisme politique et scientifique ${ }^{37}$. Il a également publié entre 1994 et 1998 la revue Racines, sous-titrée : "Racines, arts, culture et identité du nord de l'Europe ». Les objectifs de Megin s'inscrivent clairement dans la tradition völkisch du paganisme ethnopolitique et raciste. En effet, Mengal insiste sur le fait que "se connaître, c'est aussi connaître ses origines afin de se comprendre aujourd'hui et envisager les actions à venir avec plus de lucidité. La recherche des origines, c'est comme un ressourcement, c'est une clarification de la mémoire ; c'est acquérir la mémoire historique, les sens de l'histoire ; c'est retrouver ses racines, les reconnaitre et en accepter l'héritage pour forger le monde de demain ${ }^{38}$. Selon lui, « l'avenir appartient à ceux qui reprendront contact avec leur passé et qui en feront une arme psychique en vue d'une renaissance ${ }^{39}$. Parallèlement à ces activités, Mengal, fidèle à la philosophie raciale des Völkische, organise et anime des conférences, des expositions et des débats sur la généalogie, qu'il considère

34 "Entretien avec Arnaud d'Apremont », Oméga, automne 1995, s. p.

35 Ibid.

36 Le politologue, philosophe et germaniste Robert Steuckers, théoricien d'une pensée révolutionnaire conservatrice à connotation folkiste, figure parmi les animateurs importants de la Nouvelle Droite belge. Celui-ci a même remplacé Guillaume Faye comme théoricien révolutionnaire-conservateur lors du départ de ce dernier du GRECE en 1986. Il a animé ou anime les publications suivantes: Nouvelles de synergies européennes. Organe européen d'informations et d'analyses; Combat païen; Vouloir et Orientations. Lorsqu'il est parti du GRECE, Steuckers a fondé différents groupuscules dont Synergie Européenne. Dans les années 1980, les positions de Robert Steuckers, fortement influencées par le jeune Ernst Jünger et la révolution conservatrice, le situent à proximité du courant révolutionnaire conservateur.

37 Ce manifeste est reproduit in Les nouveaux païens, C. Bouchet (dir.), Coulommiers, Dualpha, 2005, p. 183-186.

38 Cité in Ialon, op. cit., p. 56.

39 Ibid. 
comme « ... un hommage rendu aux ancêtres $»^{40}$. À ce titre, il est l'administrateur d'un Service de centralisation de généalogie et de démographie de Belgique (SCGD). Il participe aussi, dans la continuité de sa pensée, à diverses associations de défense de la famille. Selon plusieurs associations antiracistes belges, Bernard Mengal se situerait dans la mouvance violente néonazie et antisémite de la Nouvelle Droite. Il a été durant un temps proche du Front national belge de Daniel Féret. Actuellement, il évolue dans la mouvance identitaire belge.

Cet activisme groupusculaire ne se résume pas à une activité intellectuelle ou du moins livresque. En effet, derrière cette production théorique, nous trouvons la volonté, chez ces militants, de mettre en place une culture qui leur serait propre et qui, surtout, leur permettrait de renouer avec leurs racines nordiques indo-européennes. Il s'agit de mettre en pratique ce néopaganisme, de lui donner une consistance sociale. Pour ce faire, ces militants se placent dans l'héritage des Völkische allemands du début du XX $\mathrm{XX}^{\mathrm{e}}$ siècle.

\section{LES PRATIQUES NÉOPAÏENNES}

Au-delà de ces présentations individuelles et groupusculaires, il faut garder à l'esprit que le néopaganisme viking est pratiqué sérieusement par ces militants. Il peut sembler folklorique à l'observateur extérieur à la fois au paganisme et à l'extrême droite, mais il fait partie de l'ontologie de ces personnes et a une fonction sociologique. Ainsi, les vieux cultes nordiques sont réactivés, ou du moins réinventés : il existe des cérémonies de mariage, de baptême, d'enterrement, soit dans des lieux fermés, soit dans la nature, mais toujours dans des propriétés privées, à l'écart du public et des curieux. Ces pratiques sont à la fois inspirées de réinventions datant de l'époque romantique et de reconstructions faites à partir des compilations de traditions populaires collectées par les folkloristes. Elles s'inspirent aussi des recherches et des découvertes des historiens et des ethnologues. Ce besoin de retrouver des racines à la fois raciales et spirituelles s'est concrétisé en France dans les années 1970 par la création d'une commission " traditions " au sein du GRECE pour aider les grécistes à célébrer différentes grandes fêtes païennes comme les solstices et les mariages, en particulier dans le cadre du domaine de Roquefavour ${ }^{41}$, animées par le néodroitier et néopaïen Maurice Rollet ${ }^{42}$.

40 "Présentation de Bernard Mengal ", Hammer Against Cross, nº 3, s. d., p. 10.

41 Anne-Marie Duranton-Crabol, Visages de la Nouvelle Droite. Le GRECE et son histoire, Paris, Presses de la Fondation nationale des sciences politiques, 1988, p. 50.

42 Mauric Rollet (1933-2014) est un militant d'extrême droite de longue date. D’abord engagé dans le combat de l'Algérie française - médecin, il a soigné des membres de l'OAS, puis par la suite Albert Spaggiari -, il fut, comme beaucoup de futurs néodroitiers, membre de la Fédération des étudiants nationalistes. Il fut l'un des membres fondateurs en 1969 du GRECE. Il était un fervent néopaïen pratiquant. 
Les textes les plus importants de cette époque restent donc les vingt-quatre numéros du bulletin de liaison de cette commission, intitulé GRECE-Traditions, qui ont été réédités en 1996 sous le titre Les traditions d'Europe ${ }^{43}$. Alain de Benoist, le principal intellectuel de la Nouvelle Droite ${ }^{44}$, a écrit $90 \%$ des textes de cette commission. La présente version, amputée de quelques articles jugés périmés, comprend en plus l'ensemble des numéros publiés postérieurement à la première édition. Il s'agit d'un manuel pour ceux qui voudraient vivre leur (nouvelle) foi.

En association avec cette spiritualité viking, les militants d'extrême droite attirés par ces pratiques développent une fascination forte pour Noël et pour le Père Noël. Ainsi, Alain de Benoist a écrit en 1982 un livre intitulé Fêter Nö̈l ${ }^{45}$. Selon Arnaud d'Apremont, faisant siennes les conclusions de la commission " Traditions » du GRECE ${ }^{46}$, le Père Noël serait un avatar du dieu Odin : " En surgissant de la nuit hivernale dans son traîneau tiré par des rennes, toutes clochettes tintinnabulantes, le Père Noël nous renvoie une image de la Chasse sauvage des anciennes nuits de Jul. Le meneur de ce cortège est le grand dieu de la tradition nordique Odin/Wotan. ${ }^{47}$ Par la suite, selon d'Apremont, la figure d'Odin se serait mélangée avec d'autres dieux païens indo-européens et pré-indo-européens, dont une divinité appelée Gorgon ou Gargan, en devenant saint Nicolas ${ }^{48}$. Le culte de saint Nicolas et de ses reliques s'acclimata en Lorraine et c'est là qu'il devint distributeur de cadeaux, une caractéristique d'Odin. Il n'est donc pas surprenant que ces néopaïens insistent sur la célébration de Noël qui « [...] représente probablement la plus grande occasion de réjouissance de l'homme moderne $»^{49}$, mais aussi « la plus vieille tradition du monde " ${ }^{50}$.

Cependant, la dérive ethniste n'est pas suivie par tous les groupes odinistes gravitant dans la nébuleuse de la Nouvelle Droite. En effet, de plus en plus de groupes reconnaissent, sous l'influence des recherches universitaires sur le monde germano-scandinave préchrétien, l'aspect chamanique, donc non indo-européen ou pré-indo-européen, du dieu Odin. Ce chamanisme est une caractéristique importante des religions nordiques préchrétiennes. Cette évolution de la vision

43 Les traditions d'Europe, A. de Benoist (dir.), Arpajon, Le Labyrinthe, 1996, n. p.

44 Alain de Benoist est un intellectuel, philosophe et journaliste de la Nouvelle Droite. Auteur prolifique, il a écrit une quarantaine d'ouvrages et plus de mille articles, portant sur l'histoire des idées, l'anthropologie, l'économie, l'écologie, etc. Il est l'auteur en 1981 d'un ouvrage important pour les militants païens d'extrême droite, Comment peut-on être païen? (Paris, A. Michel). Il n’a jamais caché sa sympathie pour le paganisme identitaire, qu'il a contribué à théoriser.

45 Alain de Benoist, Fêter Noël, Puiseaux, Pardès, 1994. Première édition : Éditions Atlas, 1982.

46 "La Saint-Nicolas : un dossier », GRECE-Traditions, no 23, 1981-1982, p. 3-19, in Les traditions d'Europe, op. cit.

47 Arnaud d'Apremont, Père Noël, op cit., p. 69.

48 Ibid., p. 50.

49 Ibid., p. 13.

50 Alain de Benoist, Fêter Noël, op. cit., quatrième de couverture. 
de la religion germano-scandinave est importante, car elle porte en elle la reconnaissance de la proximité avec les peuples sibériens de tradition religieuse chamanique, "chaman " étant d'ailleurs un terme issu d'une langue de cette région. Ainsi, l'imaginaire néonazi et racialiste du Viking " grand blond aux yeux bleus » est rejeté au profit d'une vision plus honnête de l'histoire du peuplement de l'Europe. Cependant, cette vision est toujours susceptible de développer une conception ethnocentrique, l'angle d'approche de cette élaboration doctrinale se déplaçant du racialisme vers le culturel, en promouvant la supériorité de la culture européenne, celle-ci englobant de fait les apports pré-indo-européens, par exemple, la civilisation des mégalithes et les peuples de la région circumpolaire.

Ces pratiques religieuses ont aussi pour fonction de renforcer le groupe de pratiquants, de créer une communauté. Ce dernier point est important pour comprendre la structuration mentale et le mode de fonctionnement de ces théoriciens : la famille est tout. En effet, le code de l'Odinist Fellowship à laquelle est affiliée l'association des Fils des Ases, proche de Robert Steuckers, stipule que : "1) Chaque Odiniste est le membre d'une association de parenté par l'héritage reçu des temps anciens; 2) Sois toi-même envers tes amis et parents ; Aide et soutiens le clan et la tribu partout où tu le pourras [... ; 6) Promeus et protège notre héritage spirituel ; 7) Renforce les liens de ton héritage culturel et enseigne sa valeur à tes enfants... " ${ }^{51}$. Alain de Benoist allait dans ce sens lorsqu'il écrivit, en 1977, "Vingt-cinq principes de "morale" "52 et notamment le vingt-quatrième point : "Il n’y a de piété vraie que la piété filiale, élargie aux ancêtres, à la lignée et au peuple. ${ }^{53}$ Mais, contrairement à la règle ci-dessus, il n'y a pas de référence völkisch comme le montre le dernier point : "Tous les hommes de qualité sont frères, n'importe la race, le pays et le temps. " ${ }^{54} \mathrm{Et}$, contrairement à d'autres « morales » païennes, les vingt-cinq points énoncés par Alain de Benoist relèvent avant tout de la conduite personnelle dans la société, définition de la «morale », et non de la règle communautaire.

C'est en Belgique, dans l'espace flamand, que nous trouvons le meilleur exemple de vie communautaire païenne. Depuis 1993, il existe à Anvers une association du nom de Traditie (Tradition), d'orientation païenne. Son responsable est Peter Cineger, un militant du parti d'extrême droite Vlaamse Volksbeweging (Mouvement populaire flamand ou VVB), un groupuscule régionaliste

51 Gjallarhorn, no 5, mai 1999. Supplément de Megin, réservé aux abonnés de Megin. Cité in Ialon, $\mathrm{n}^{\circ} 12$, s. d. (1999 ?), p. 43. C’est nous qui soulignons. Ces revues étaient des publications belges d'idéologie révolutionnaire-conservatrice, héritières des groupes révolutionnaires-conservateurs allemands de la république de Weimar.

52 Alain de Benoist, "Vingt-cinq principes de morales ", Les idées à l'endroit, Paris, Éditions libres Hallier, 1979, p. 49-54.

53 Ibid., p. 53.

54 Ibid., p. 54. 
fondé en 1953 par deux anciens collaborateurs et qui vise à défendre les intérêts de la cause flamande. Traditie est liée à la fondation Delta, Deltapers, dirigée par le représentant belge de la Nouvelle Droite Luc Pauwels. Selon l'un de ses animateurs, Koenraad Logghe, le choix de la pratique cultuelle s'est fait par vote. L'association a choisi l'Ásatrú " parce que cette religion est reconnue [...] et parce qu'elle contient le moins de déviations pseudo-religieuses, comme c'est souvent le cas dans les groupes se réclamant du néo-paganisme " ${ }^{55}$. Selon Koenraad Logghe, " ... il existe [en Flandre] une longue tradition vivante de fêtes traditionnelles [c'est-à-dire païennes] : les solstices, les fêtes de mai, de la moisson, etc. font depuis de longues années partie du cycle annuel en Flandre $"^{56}$. Traditie dispose d'une structure d'enseignement secondaire, ayant son propre cursus d'éducation religieuse : "Nous avons obtenu du ministre de l'Éducation un accord pour une dispense des cours de religion ou de morale laïque ; pendant ces heures "libres", les enfants peuvent étudier leur propre "religion traditionnelle" à condition de disposer de cours adéquats. Ces cours sont prêts et traitent d'archéologie et d'histoire, de symbolique et d'art, de philosophie, de mythes, d'écologie, d'éthique, de fêtes, de relations, de généalogie et ce, pour trois niveaux (13 à 14 ans, 15 à 16 ans et 17 à 18 ans). Jusqu’à présent [en 1997] une dizaine de familles a déjà obtenu la dispense. $"{ }^{57}$ Ces personnes vivent quasiment en mode clos, sur le modèle des communautés païennes américaines. Elles se placent également dans la tradition des communautés völkisch allemandes du début du XX ${ }^{e}$ siècle.

L'attrait pour l'imaginaire viking se manifeste également d'une autre façon dans ces milieux. Les générations les plus jeunes aiment écouter une scène musicale née dans les années 1980 en Norvège et se réclamant de l'extrême droite : le NSBM (National-Socialist Black Metal), une musique violente et très rythmée. L'un de ses plus grands représentants vit aujourd'hui en France depuis sa sortie de prison : Kristian "Varg» (loup en norvégien) Vikernes. Cet ancien skinhead est le leader du groupe Burzum. Il a purgé une peine de prison de vingt et un ans pour meurtre et destructions d'églises. Misanthrope, sataniste, il a évolué depuis son arrestation vers une forme d'odinisme raciste, mâtiné d' "occultisme nazi " ${ }^{58}$. Il appartient, ou a appartenu, à plusieurs structures néopaïennes völkisch comme le Front païen

55 "À la recherche du Graal. Entretien avec Koenraad Logghe », Antaïos, no 12, hiver 1997, p. 34. Cette revue a été fondée et animée par le néodroitier belge, et militant païn, Christopher Gérard, qui s'est inspiré de la revue éponyme fondée par Ernst Jünger et Mircea Eliade et publiée en 1959 et 1971.

56 Ibid., p. 34.

57 Ibid.

58 Charles Morrow, "Resurgent Atavism ? Resurgent Nazism, Or, Wotan Made Me Do It ", in Soundtracks to the White Revolution. White Supremacist Assaults on Youth Music Subcultures, D. Burghart (dir.), Chicago, Center for New Community, 1999, p. 68-70. Voir aussi Encyclopedia of White Power. A Sourcebook of the Radical, Racist Right, J. Kaplan (dir.), Walnut Creek, Altamira Press, 2000 ; The Cultic Milieu. Oppositional Subcultures in the Age of Globalisation, J. Kaplan et H. Lööw (dir.), Walnut Creek, Altamira Press, 2002. 
norvégien (Norsk Hedensk Front) et le Front païen germanique (Allgermanische Heidnische Front). Aujourd'hui, il se réclame du paganisme nordique. Il est aussi l'un des membres fondateurs du mouvement extrémiste norvégien Résistance aryenne (Hvit Arisk Motstand). Depuis son incarcération, il a publié plusieurs essais politico-raciologiques, parus dans des revues underground, dont Filosofem, une revue dirigée par l'un de ses proches, Vidar von Herske, lui aussi membre de Burzum. Parallèlement à cette activité, Vikernes a continué de produire de la musique et affiché son soutien à un groupuscule néonazi, l'" Einsatzgruppe ", qui prévoyait de commettre des attentats en Norvège. En 2011, Vikernes soutint Marine Le Pen et le Front national français. Militant survivaliste, vivant à la campagne, il a été brièvement arrêté par la gendarmerie pour possession d'armes de guerre. Apôtre de la mixophobie, il défend une séparation physique des groupes ethniques, prônant la création de "colonies " blanches dans des zones reculées. Passionné par le paganisme viking, omniprésent dans son œuvre musicale, Vikernes a fait paraître en 2016 une traduction française de l'un de ses ouvrages sur les mythes scandinaves ${ }^{59}$. Son aspect physique a changé durant cette évolution spirituelle : du "look» typique du Black Metal (cheveux longs, maquillage, vêtement de cuir, etc.), il est passé au « look » völkisch (barbe, cheveux courts, vêtements de laine inspirés des vêtements vikings, etc.). Plus largement, ces groupes prônent l'avènement d'un "Empire païen aryen ${ }^{60} \ldots$ Néanmoins, le recours aux thèmes nordicistes et au paganisme viking reste très superficiel et se cantonne le plus souvent à des titres de chansons ou d'albums ou à l'esthétique des pochettes.

\section{UN ETHNOCENTRISME ${ }^{61}$ AFFIRMÉ}

Quoi qu'il en soit, l'intérêt pour le monde et la religion vikings est la manifestation d'un ethnocentrisme affirmé. Si les milieux étudiés ici participent à une nouvelle étape de la circulation de l'imaginaire viking, les militants ont des points communs avec leurs prédécesseurs de la première moitié du XX $\mathrm{XX}^{\mathrm{e}}$ siècle. L'ethnocentrisme assumé en fait partie. En effet, ces militants d'extrême droite voyaient, et voient toujours, dans ces Vikings des créateurs de civilisations, reformulant le très raciste discours de la race blanche civilisatrice, guidant les races inférieures, mais d'un usage moins connoté que la thèse aryenne de triste mémoire, qu'ils évitent, pour la plupart, de citer. Le spécialiste du monde scandinave Régis Boyer, non sans raison, se montre d'ailleurs très sévère au sujet $\mathrm{du}$ " surhomme aryen » qu'il associe logiquement à la figure fantasmée du «Viking » : « ... Ex septentrione

59 Varg Vikernes, Magie et religion en Scandinavie antique, Paris, Rubicon, 2016.

60 Voir Stéphane François, Au-delà des vents du Nord, op. cit., p. 247-260.

61 Nous entendons ici par "ethnocentrisme " les discours qui : 1/promeuvent une surévaluation de la société d'appartenance de celui qui l'énonce ; 2/dévalorisent les autres cultures ou civilisations ; 3 / expriment des formes extrêmes d'intolérance (culturelle, religieuse, politique), parfois sous des formes subtiles. 
lux, et vous savez très bien comme moi les théories dangereuses qui viennent de là : du nord, vient la lumière, le Surhomme vient du nord, l'Aryen vient du nord, etc., nous avons déjà abordé ce sujet dont je ne parviens pas à comprendre qu'il trouve toujours, aujourd'hui, des adeptes parfois instruits, sortes de nostalgiques crispés sur un passé illusoire et refusant contre tout bon sens les tendances de la modernité. ${ }^{62}$ Les militants d'extrême droite lisent les universitaires, mais refusent leurs conclusions. Bernard Mengal rejette les positions de Régis Boyer : "Monsieur Régis Boyer a fait un travail remarquable en traduisant en français des textes norrois et en les commentant abondamment. Nous lui sommes reconnaissants pour son œuvre de grande valeur scientifique. En revanche, nous ne le suivons pas dans certaines de ses conclusions. [...] Hélas! Pour monsieur Boyer, en dépit de toute son érudition, force est de constater qu'il se soumet aux satrapes du moment. Ce faisant, il trahit l'esprit des Vikings qui, quoi qu'il en dise, étaient avant tout des guerriers intrépides et non pas des marchands bornés et hypermatérialistes ... ${ }^{63}$.

Guerriers intrépides donc, marins aventureux, les Vikings auraient créé, selon ces militants d'extrême droite, les civilisations précolombiennes, reprenant les thèses du théoricien raciste Jacques de Mahieu ${ }^{64}$. Cette affirmation se fondait sur des « recherches archéologiques » qu'il avait menées en Amérique du Sud. Mahieu reprenait l'idée, très classique dans certains milieux de l'extrême droite nordiciste, selon laquelle des populations indo-européennes, qu'il appelle " Troyens " dans quelques textes ${ }^{65}$, aient pu se réfugier dans ce continent et civiliser les populations locales. Il publia ainsi une quinzaine d'ouvrages, traduits en plusieurs langues (français, espagnol, allemand, italien, anglais) sur cette question, qui eurent un succès éditorial. Les livres les plus importants ont été traduits en français entre 1971 et 1980 : Le grand voyage du Dieu Soleil (1971) aux Éditions et publications premières ; L'agonie du Dieu Soleil. Les Vikings en Amérique du Sud (1974) et Les Templiers en Amérique (1980), chez Robert Laffont ; Drakkars sur l'Amazone (1977) et L'imposture de Colomb. La géographie sacrée de l'Amérique (1979), chez Copernic. Tous ces ouvrages cherchent à démontrer le rôle civilisateur des Vikings

62 Ibid., p. 195.

63 «Entretien avec Bernard Mengal », Hammer Against Cross, no 3, s. d., p. 15.

64 Jacques-Marie de Mahieu est un personnage méconnu et très atypique. On ne connaît en France que des aspects parcellaires de sa vie, bien qu'il ait connu des succès éditoriaux dans les années 1970. Pour les uns, il s'agit d'un théoricien raciste du péronisme, voire d'un raciologue nazi. Pour les autres, c'est un archéologue ayant mis à jour la présence de Vikings en Amérique latine (Jacques de Mahieu, «Les indiens blancs du Paraguay ", Nouvelle École, no 24, hiver 1973-1974, p. 46-59). La réalité est plus complexe et surtout moins tranchée. En effet, Jacques de Mahieu était à la fois un raciologue français, proche du négationniste suisse Gaston-Armand Amaudruz, et un auteur à succès de ce qu'on appelle communément l'« histoire mystérieuse ». Il fut aussi un passeur d'idées entre différents milieux et différentes cultures.

65 Jacques de Mahieu, La fabuleuse épopée des Troyens en Amérique du Sud, Puiseaux, Pardès, 1998. 
dans la naissance de civilisations complexes sur le continent latino-américain et à expliquer la construction de monuments cyclopéens par une présence «blanche». Cet ethnocentrisme fut condamné pour son évident racialisme et tourné en dérision par les spécialistes de la question. Cela a été d'autant plus facile que Mahieu, à la même époque, publiait des ouvrages de raciologie, comme son Précis de biopolitique, paru en $1969^{66}$ chez un éditeur canadien proche du mouvement néonazi et négationniste Nouvel ordre européen (NOE). D’ailleurs, Jacques de Mahieu, docteur honoris causa en médecine d'une université argentine, était le fondateur, cette même année, avec les néonazis et négationnistes Gaston-Armand Amaudruz $^{67}$ et le docteur Jacques Baugé-Prévost ${ }^{68}$ (membres du NOE), d'un Institut supérieur des sciences psychosomatiques, biologiques et raciales, créé à Barcelone et basé à Montréal. En raison de ses « connaissances " raciologiques, il fut nommé directeur du Département d'anthropologie de l'université de Buenos Aires. Ses " recherches ", dans ce domaine, consistaient avant tout en une étude physique des corps de peuples indigènes (longueur des membres, morphologie, physionomie, forme du crâne, traits du visage, couleur de la peau, des cheveux et des yeux, pilosité, etc.), ainsi que l'étude sérologique des groupes observés. Elles comprenaient aussi une crâniométrie de ces populations (indice céphalique) ${ }^{69}$. Le but de ces observations raciales était de prouver la dégénérescence de ces descendants de métis de Vikings et d'Indiens, principalement Guayakis, qu'il appelait les «Indiens blancs du Paraguay » et de montrer implicitement les ravages du métissage sur les groupes " purs ", ces groupes vikings qui commirent selon lui le crime de s'accoupler avec des races inférieures, en l'occurrence autochtones.

Les thèses de Mahieu furent reprises dans les années 1970 par la frange la plus radicale de l'extrême droite française, dont Jean-Claude Valla, membre fondateur du GRECE proche des négationnistes ${ }^{70}$. Selon son camarade Michel Marmin,

66 Jacques de Mahieu, Précis de biopolitique, Montréal, Éditions celtiques, 1969.

67 Gaston-Armand Amaudruz est un vieux militant nazi (il est né en 1920) et négationniste suisse. Ses livres sont violemment antisémites et racistes. Il a fondé en 1951 avec l'ex-SS français René Binet, le Nouvel ordre européen (NOE), qui a été remplacé en 2013 par l'Action européenne. Sur Gaston-Armand Amaudruz et ses idées, voir Damir Skenderovic, Luc van Dongen, "Gaston-Armand Amaudruz, pivot et passeur européen ", in Doctrinaire, vulgarisateurs et passeurs des droites radicales au XX' siècle (EuropeAmériques), O. Dard (dir.), Berne, Peter Lang, 2012, p. 211-230.

68 Le docteur Jacques Baugé-Prévost est un naturopathe québécois, membre du NOE. Outre ses livres sur la naturopathie, il est l'auteur d'une Éthique biologique de l'homme blanc, paru initialement à Montréal aux Éditions celtiques en 1965, réédité en 2018 par les Éditions du Lore, un éditeur identitaire néonazi.

69 Jacques de Mahieu, "Les indiens blancs du Paraguay ", op. cit.

70 Jean-Claude Valla (1944-2010), ancien militant de la FEN et d'Europe-Action, est une figure importante de la Nouvelle Droite. Il quitte le GRECE en 1986 tout en restant proche. Journaliste (il a été rédacteur en chef dans les années 1970 de Valeurs actuelles puis d'Éléments), il a été le premier directeur de la publication du Figaro Magazine. Il a aussi écrit des livres d'histoire, surtout sur la Seconde Guerre mondiale et la Collaboration avec « Les Cahiers libres d'histoire». 
"Jean-Claude Valla, ce n'est pas faire injure à sa mémoire, avait la passion de l'histoire secrète, celle que l'on nous cache derrière l'histoire "officielle". [...] Jean-Claude s'était ainsi convaincu que la civilisation des Incas était le fruit d'une colonisation du Pérou par les Vikings et que leur organisation sociale était parfaitement représentative de la trifonctionnalité indo-européenne $»^{71}$. En effet, Jean-Claude Valla développa cette idée dans un ouvrage publié en 1976 chez un éditeur genevois, Famot, La civilisation des Incas. Ces thèses furent aussi défendues à cette époque par Michel Marmin qui signa en 1977 un article intitulé "Les Vikings en Amérique du Sud ?" dans Éléments ${ }^{72}$. Alain de Benoist cita aussi Jacques de Mahieu en 1977 comme référence scientifique dans sa notice sur « Les Vikings en Amérique ", publiée dans son $V u$ de droite ${ }^{73}$ - mais il est vrai que l'idée d'une présence viking en Amérique était ancienne sous la plume d'Alain de Benoist : il la soutenait déjà en 1964 dans un article publié dans Europe-Action ${ }^{74}$, et continua de défendre ces thèses tout au long des années 1970. C'est d'ailleurs lui qui fit entrer Jacques de Mahieu dans le comité de patronage de Nouvelle École.

Cet ethnocentrisme, digne de la première moitié du XXe siècle, est une composante importante du néopaganisme nordique, encore aujourd'hui, surtout dans les milieux les plus radicaux. En effet, ceux-ci ne masquent pas leur filiation avec les théoriciens du racisme ainsi qu’avec les Völkische allemands des années 1920 et 1930. Simplement, comme nous l'avons dit en introduction de cet article, ils ont remplacé le paganisme germanique, ou plus précisément aryen, du début $\mathrm{du} \mathrm{XX}^{\mathrm{e}}$ siècle par un néopaganisme d'inspiration viking moins connoté. Cette réhabilitation s'est faite dans un premier temps en évitant les références trop marquées. Ainsi, Jean Mabire fit l'éloge de Nicolai Frederik Severin Grundtvig ${ }^{75}$ qui décida de vulgariser la mythologie scandinave, permettant ainsi la redécouverte de ces mythes et l'essor de groupes païens scandinaves à compter de la fin du $\mathrm{XIX}^{\mathrm{e}}$ siècle. Ces militants d'extrême droite reprirent aussi aux premières communautés païennes leurs pratiques sociales, qu’ils ont enrichies des découvertes scientifiques et ethnologiques récentes. L'autre grande nouveauté de ces militants néopaïens, y compris pour les plus radicaux, a été la mise en avant d'un ethnodifférentialisme radical, promouvant officiellement la différence et la préservation des identités, non sans ambiguïté : dès 1981, l'universitaire britannique Martin Barker y voyait, à juste raison d'ailleurs, la manifestation d'une nouvelle

71 Michel Marmin, "Avec Jean-Claude Valla au Chili du général Pinochet ", Eléments, juilletseptembre 2010, p. 60.

72 Michel Marmin, "Les Vikings en Amérique du Sud ? ", Éléments, no 21-22, avril-juillet 1977.

73 Alain de Benoist, "Les Vikings en Amérique ", Vu de Droite. Anthologie critique des idées contemporaines, Paris, Labyrinthe, 2001, p. 68-77.

74 Fabrice Laroche, «Les Viking en Amérique », Europe-Action, n 15, mars 1964, p. $25-26$.

75 Jean Mabire, Les grands aventuriers de l'histoire. Les éveilleurs de peuples, Paris, Fayard, 1982. 
forme de racisme ${ }^{76}$, fondé sur l'incompatibilité des cultures. Quoi qu'il en soit, ces militants d'extrême droite semèrent dès la fin des années 1970 des graines, idéologiques et religieuses, qui grandirent et se diffusèrent au sein des extrêmes droites européennes et américaines. Aujourd'hui, le néopaganisme en est une des spiritualités les plus importantes, la figure mythique du Viking fascinant toujours autant depuis Arthur de Gobineau, à l'origine, avec Le roman de Manfrédine ${ }^{77}$, de la figure du Viking comme représentation du surhomme.

76 Martin Barker, The New racism. Conservatism and the Ideology of the Tribe, Londres, Junction Books, 1981.

77 Régis Boyer, Le mythe viking dans les lettres françaises, Paris, Porte-Glaive, 1990, p. 136. 